\title{
Dynamic Geometry of Cubic Polynomial
}

\author{
Gagik Aghekyan', Karen Sahakyan,"* \\ ${ }^{1}$ Department of Applied Mathematics and Computer Science of Russian-Armenian University, Yerevan, Armenia \\ ${ }^{2}$ Department of Mathematics and Mechanics of Yerevan State University, Yerevan, Armenia \\ *Corresponding Author: karen_sahakyan@mail.ru
}

Copyright $(2013$ Horizon Research Publishing All rights reserved.

\begin{abstract}
In the paper we consider the dynamic behavior of the critical points of some cubic polynomials, with the motion of one of the roots of the polynomial along a given trajectory.

Some dynamic property of polynomials is investigated. The statements about traces of critical points of some polynomials are proved. The equations of curves, on which critical points move, are obtained.
\end{abstract}

Keywords Geometry of Polynomials, Zeros of Polynomials, Critical Points

\section{Introduction}

In 1836 , Gauss showed that all the roots of $P^{\prime}$, distinct from the multiple roots of the polynomial $P$ itself, serve as the points of equilibrium for the field of forces created by identical particles placed at the roots of $P$ (provided that $r$ particles are located at the root of multiplicity $r$ ). The following equality to zeros provides a quick proof of Gauss-Lucas theorem (see for example [1] or [2]). Thus was appeared the branch of mathematics, which after the book of Morris Marden [3], was called Geometry of Polynomials. The polynomial conjectures of Sendov and Smale are two challenging problems of this branch $[4,5,6]$.

One of the beautiful theorems of mathematics is Marden's theorem $[3,7]$. It gives a geometric relationship between the zeros of a third-degree polynomial with complex coefficients and the zeros of its derivative. A more general version of this theorem, due to Linfield [8].

This article focuses on the dynamic behavior of critical points in the case of moving one of the roots of cubic polynomial on a given trajectory. The equations of the curves, where the critical points move, are obtained. Discovered new geometric properties of positions of the zeros and critical points of a complex polynomial of degree three. The case of multiple roots of the given polynomial is considered as well.

\section{The Case of Simple Roots}

Suppose there are three points $z_{1}=0, z_{2}=1$ and $z_{3}=e^{\llbracket \varphi}$ where the angle $\varphi$ varies from 0 to $2 \pi$. Then the polynomial

$$
P(z)=z(z-1)\left(z-e^{i \varphi}\right)=z^{3}-\left(1+e^{i \varphi}\right) z^{2}+e^{i \varphi} z
$$

is a polynomial with roots $z_{1}, z_{2}$ and $z_{3}$.

Then the first derivative is

$$
P^{\prime}(z)=3 z^{2}-2\left(1+e^{i \varphi}\right) z+e^{i \varphi}
$$

Theorem 1. Let $z_{1}=0, z_{2}=1$ and $z_{3}=e^{i \varphi} \quad$ where the angle $\varphi$ varies from 0 to $2 \pi$, are roots of a complex polynomial of the third degree. Then a geometrical place of critical points of this polynomial consists of two circles given by the equations:

$$
|z|=\frac{1}{\sqrt{3}}
$$

and

$$
\left|z-\frac{2}{3}\right|=\frac{1}{3}
$$

Proof. The zeros of $P^{\prime}$ are given by the formula

$$
z_{1,2}=\frac{1}{3}\left(1+e^{i \varphi} \pm \sqrt{1+e^{2 i \varphi}-e^{i \varphi}}\right)
$$

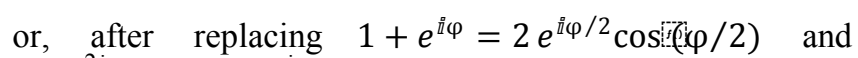
$1+e^{2 i \varphi}=2 \cos \varphi e^{i \varphi}$,

$$
z_{1,2}=\frac{1}{3}\left(2 \cos \frac{\varphi}{2} e^{i \varphi / 2} \pm \sqrt{e^{i \varphi}(2 \cos \varphi-1)}\right)
$$

Depending on whether $\varphi \in\left(\frac{\pi}{3}, \frac{5 \pi}{3}\right)$ or $|\varphi| \leq \frac{\pi}{3}$, we write

$$
z_{1,2}=\frac{e^{i \varphi / 2}}{3}\left(2 \cos \frac{\varphi}{2} \pm i \sqrt{1-2 \cos \varphi}\right)
$$

or 


$$
z_{1,2}-\frac{2}{3}=\frac{e^{i \varphi / 2}}{3}\left(2 i \sin \frac{\varphi}{2} \pm i \sqrt{2 \cos \varphi-1}\right)
$$

In the first case we have

$$
\begin{aligned}
& \left|z_{1,2}\right|=\frac{1}{3} \sqrt{4 \cos ^{2} \frac{\varphi}{2}+1-2 \cos \varphi}= \\
& \frac{1}{3} \sqrt{2 \cos \varphi+2+1-2 \cos \varphi}=\frac{1}{\sqrt{3}}
\end{aligned}
$$

while in the second case we get

$$
\left|z_{1,2}\right|=\frac{1}{3} \sqrt{4 \sin ^{2} \frac{\varphi}{2}+2 \cos \varphi-1}=\frac{1}{3}
$$

Which prove this theorem.

Theorem 2. Geometric place of critical points of the polynomial $P(z)$ is the intersection of circle (4) and the straight line

$$
y=\operatorname{tg} \frac{\varphi}{2} x, \text { when }|\varphi| \leq \frac{\pi}{3}
$$

and the intersection of circle (3) and the straight line

$$
y=\operatorname{ctg} \frac{\varphi}{2}\left(\frac{2}{3}-x\right), \text { when } \frac{\pi}{3}<\varphi<\frac{5 \pi}{3}
$$

Proof. Using the formulas

$$
z_{1,2}-\frac{2}{3}=\frac{e^{i \varphi / 2}}{3}\left(2 i \sin \frac{\varphi}{2} \pm i \sqrt{2 \cos \varphi-1}\right)
$$

and

$$
z_{1,2}=\frac{e^{i \varphi / 2}}{3}\left(2 \cos \frac{\varphi}{2} \pm i \sqrt{1-2 \cos \varphi}\right)
$$

from the previous proving we obtain $\frac{\operatorname{Im} z_{1,2}}{\operatorname{Re} z_{1,2}}=\operatorname{tg} \frac{\varphi}{2}$, and $\frac{\operatorname{Im} z_{1,2}}{\frac{2}{3}-\operatorname{Re} z_{1,2}}=\operatorname{ctg} \frac{\varphi}{2} \quad$ accordingly, which prove this theorem.

Remark 1. Geometric place the solutions of equation $P^{\prime \prime}(z)=0$, when $\varphi$ varies from 0 to $2 \pi$, consists of the circle given by the equation $\left|z-\frac{1}{3}\right|=\frac{1}{3}$. And for given $\varphi$ this solution is the intersection of its circle with the straight line $y=\operatorname{tg} \frac{\varphi}{2} x$.

The proof is trivial.

Theorem 3. Suppose that $z_{1}=0$ and $z_{2}=1$ are two fixed roots of a cubic polynomial $P(z)$ and the third root $z_{3}$, moves along the perpendicular bisector of the segment $[0,1]$, then

a) The geometric locus of critical points of $P(z)$, for $z_{3}$ such that $\left|\operatorname{Im} z_{3}\right| \leq \frac{\sqrt{3}}{2}$, is the circle

$$
\left(x-\frac{1}{2}\right)^{2}+y^{2}=\left(\frac{\sqrt{3}}{6}\right)^{2}
$$

which intersection with a straight line $y=\frac{\operatorname{Im} z_{3}}{3}$ determines the location of critical points of $P(z)$. b) For $z_{3}$ such that $\left|\operatorname{Im} z_{3}\right|>\frac{\sqrt{3}}{2}$, the geometric locus of critical points of $P(z)$ is the perpendicular bisector of the segment $[0,1]$. Which intersection with a circle, the center of which divides the segment $\left|z_{1}-z_{3}\right|$ in the ratio of 1:3 measured from the point of $z_{1}$, with a radius equal to $\frac{\left|z_{1}-z_{3}\right|}{3}$ determine the location of critical points of $P(z)$.

Proof. Let $P(z)$ is cubic polynomial with roots $z_{1}=0$, $z_{2}=1$ and $z_{3}=\frac{1}{2}+i y_{3}$.

Then

$$
\begin{array}{r}
P(z)=z(z-1)\left(z-\left(\frac{1}{2}+i y_{3}\right)\right) \\
\text { or } P(z)=z^{3}-\left(\frac{3}{2}+i y_{3}\right) z^{2}+\left(\frac{1}{2}+i y_{3}\right) z .
\end{array}
$$

And

$$
P^{\prime}(z)=3 z^{2}-\left(3+2 i y_{3}\right) z+\left(\frac{1}{2}+i y_{3}\right)
$$

a) Solving the equation $P^{\prime}(z)=0$, with the condition $\left|y_{3}\right| \leq \frac{\sqrt{3}}{2}$, we get

$$
\xi=\frac{1}{2} \pm \sqrt{\frac{1}{12}-\frac{y_{3}^{2}}{9}}+i \frac{y_{3}}{3}
$$

hence $\left|\xi-\frac{1}{2}\right|=\frac{\sqrt{3}}{6}$ and $\operatorname{Im} \xi=\frac{y_{3}}{3}$, which proves the statement a) of this theorem. 
b) Solving the equation $P^{\prime}(z)=0$, with the condition $\left|y_{3}\right|>\frac{\sqrt{3}}{2}$, we get

$$
\xi=\frac{1}{2}+i\left(\frac{y_{3}}{3} \pm \sqrt{\frac{y_{3}^{2}}{9}-\frac{1}{12}}\right)
$$

hence $\operatorname{Re} \xi=\frac{1}{2}$ and

$$
\left|\xi-\left(\frac{1}{6}+i \frac{y_{3}}{3}\right)\right|=\sqrt{\frac{1}{36}+\frac{y_{3}^{2}}{9}}=\frac{\left|z_{1}-z_{3}\right|}{3}
$$

Which proves the statement $b$ ) of this theorem.

\section{The Case of Multiple Roots}

Suppose there are three points $z_{1}=0, z_{2}=1$ and $z_{3}=e^{i \varphi}$ where the angle $\varphi$ varies from 0 to $2 \pi$. Then the polynomial

$$
P(z)=z^{\alpha}(z-1)^{\beta}\left(z-e^{i \varphi}\right)^{\gamma}
$$

is a polynomial with multiple roots $z_{1}, z_{2}$ and $z_{3}$ where $\alpha, \beta, \gamma$ natural numbers with are $\alpha+\beta+\gamma>3$.

Then the first derivative is

$$
\begin{aligned}
& P^{\prime}(z)=z^{\alpha-1}(z-1)^{\beta-1}\left(z-e^{i \varphi}\right)^{\gamma-1} \times \\
& \left((\alpha+\beta+\gamma) z^{2}-\left(\alpha e^{i \varphi}+\alpha+\beta e^{i \varphi}+\gamma\right) z+\alpha e^{i \varphi}\right)
\end{aligned}
$$

Then the simple zeros of $P^{\prime}(z)$ will be given by the formula

$$
\begin{aligned}
& z_{1,2}=\frac{\left((\alpha+\beta) e^{i \varphi}+(\alpha+\gamma)\right)}{2(\alpha+\beta+\gamma)} \pm \\
& \frac{\sqrt{(\alpha+\beta)^{2} e^{2 i \varphi}-2\left(\alpha^{2}+\alpha \beta+\alpha \gamma-\beta \gamma\right) e^{i \varphi}+(\alpha+\gamma)^{2}}}{2(\alpha+\beta+\gamma)}
\end{aligned}
$$

in the case when $\beta=\gamma$ will be

$$
\begin{aligned}
& z_{1,2}=\frac{(\alpha+\beta)\left(e^{i \varphi}+1\right)}{2(\alpha+2 \beta)} \pm \\
& \frac{\sqrt{(\alpha+\beta)^{2}\left(e^{2 i \varphi}+1\right)-2\left(\alpha^{2}+2 \alpha \beta-\beta^{2}\right) e^{i \varphi}}}{2(\alpha+2 \beta)}
\end{aligned}
$$

or, after replacing $1+e^{i \varphi}=2 \cos \frac{\varphi}{2} e^{i \varphi / 2}$

and $1+e^{2 i \varphi}=2 \cos \varphi e^{i \varphi}$,

$$
\begin{gathered}
z_{1,2}=\frac{(\alpha+\beta) 2 \cos \frac{\varphi}{2} e^{i \varphi / 2}}{2(\alpha+2 \beta)} \pm \\
\frac{\sqrt{(\alpha+\beta)^{2} 2 \cos \varphi e^{i \varphi}-2\left(\alpha^{2}+2 \alpha \beta-\beta^{2}\right) e^{i \varphi}}}{2(\alpha+2 \beta)}
\end{gathered}
$$

And depending on $\varphi$ we obtain

$$
\begin{aligned}
& z_{1,2}=\frac{(\alpha+\beta) e^{i \varphi / 2}}{2(\alpha+2 \beta)} \times \\
& \left(2 \cos \frac{\varphi}{2} \pm i \sqrt{\frac{2\left(\alpha^{2}+2 \alpha \beta-\beta^{2}\right)}{(\alpha+\beta)^{2}}-2 \cos \varphi}\right)
\end{aligned}
$$

when $\cos \varphi-\frac{\alpha^{2}+2 \alpha \beta-\beta^{2}}{(\alpha+\beta)^{2}}<0$

$\left(\arccos \frac{\alpha^{2}+2 \alpha \beta-\beta^{2}}{(\alpha+\beta)^{2}}<\varphi<2 \pi-\arccos \frac{\alpha^{2}+2 \alpha \beta-\beta^{2}}{(\alpha+\beta)^{2}}\right)$

and

$$
\begin{aligned}
& z_{1,2}=\frac{(\alpha+\beta) e^{i \varphi / 2}}{2(\alpha+2 \beta)} \times \\
& \left(2 \cos \frac{\varphi}{2} \pm \sqrt{2 \cos \varphi-\frac{2\left(\alpha^{2}+2 \alpha \beta-\beta^{2}\right)}{(\alpha+\beta)^{2}}}\right)
\end{aligned}
$$

when $\cos \varphi-\frac{\alpha^{2}+2 \alpha \beta-\beta^{2}}{(\alpha+\beta)^{2}} \geq 0$,

$\left(|\varphi| \leq \arccos \frac{\alpha^{2}+2 \alpha \beta-\beta^{2}}{(\alpha+\beta)^{2}}\right)$.

Theorem 4. The geometric place of critical points of polynomial $P(z)=z^{\alpha}(z-1)^{\beta}\left(z-e^{i \varphi}\right)^{\gamma}$, where the angle $\varphi$ varies from 0 to $2 \pi$, consists of two circles given by the equations:

$$
|z|=\sqrt{\frac{\alpha}{\alpha+2 \beta}}
$$

and

$$
\left|z-\frac{\alpha+\beta}{\alpha+2 \beta}\right|=\frac{\beta}{\alpha+2 \beta}
$$

Proof. From (12) we obtain

$\left|z_{1,2}\right|=$ 


$$
\begin{aligned}
& \frac{(\alpha+\beta)}{2(\alpha+2 \beta)} \sqrt{4 \cos ^{2} \frac{\varphi}{2}+\frac{2\left(\alpha^{2}+2 \alpha \beta-\beta^{2}\right)}{(\alpha+\beta)^{2}}-2 \cos \varphi}= \\
& =\frac{(\alpha+\beta)}{2(\alpha+2 \beta)} \sqrt{2+\frac{2\left(\alpha^{2}+2 \alpha \beta-\beta^{2}\right)}{(\alpha+\beta)^{2}}}= \\
& =\frac{1}{2(\alpha+2 \beta)} \sqrt{4 \alpha^{2}+8 \alpha \beta}=\sqrt{\frac{\alpha}{\alpha+2 \beta}}
\end{aligned}
$$

And from (13)

$$
\begin{gathered}
\left|z_{1,2}-\frac{\alpha+\beta}{\alpha+2 \beta}\right|=\frac{(\alpha+\beta)}{2(\alpha+2 \beta)} \times \\
\sqrt{4 \sin ^{2} \frac{\varphi}{2}+2 \cos \varphi-\frac{2\left(\alpha^{2}+2 \alpha \beta-\beta^{2}\right)}{(\alpha+\beta)^{2}}}= \\
=\frac{(\alpha+\beta)}{2(\alpha+2 \beta)} \sqrt{2-\frac{2\left(\alpha^{2}+2 \alpha \beta-\beta^{2}\right)}{(\alpha+\beta)^{2}}}=\frac{\beta}{\alpha+2 \beta}
\end{gathered}
$$

Which prove this theorem.

Theorem 5. Geometric place of critical ${ }_{\beta}$ points of the polynomial $P(z)=z^{\alpha}(z-1)^{\beta}\left(z-e^{i \varphi}\right)^{\beta}$ is the intersection of circle (15) and the straight line

$$
y=\operatorname{tg} \frac{\varphi}{2} x \text {, when }|\varphi| \leq \arccos \frac{\alpha^{2}+2 \alpha \beta-\beta^{2}}{(\alpha+\beta)^{2}}
$$

and the intersection of circle (14) and the straight line

$$
\begin{aligned}
& y=\operatorname{ctg} \frac{\varphi}{2}\left(\frac{\alpha+\beta}{\alpha+2 \beta}-x\right), \text { when } \\
& \arccos \frac{\alpha^{2}+2 \alpha \beta-\beta^{2}}{(\alpha+\beta)^{2}}<\varphi<2 \pi-\arccos \frac{\alpha^{2}+2 \alpha \beta-\beta^{2}}{(\alpha+\beta)^{2}}
\end{aligned}
$$

Proof. Using the formulas (13) and (12) we obtain $\frac{\operatorname{Im} z_{1,2}}{\operatorname{Re} z_{1,2}}=\operatorname{tg} \frac{\varphi}{2} \quad$ and $\quad \frac{\operatorname{Im} z_{1,2}}{\frac{\alpha+\beta}{\alpha+2 \beta}-\operatorname{Re} z_{1,2}}=\operatorname{ctg} \frac{\varphi}{2}$ accordingly, which prove this theorem.

Remark 2. Geometric place the solutions of equation $P^{\prime \prime}(z)=0, \varphi$ varies from 0 to $2 \pi$, consists of the circle given by the equation $\left|z-\frac{\alpha+\beta}{2(\alpha+2 \beta)}\right|=\frac{\alpha+\beta}{2(\alpha+2 \beta)}$. And for given $\varphi$ this solution is the intersection of its circle with the straight line $y=\operatorname{tg} \frac{\varphi}{2} x$.

The proof is trivial.

Theorem 6. Suppose that $z_{1}=0$ and $z_{2}=1$ are two fixed roots of polynomial $P(z)$ of multiplicity $m$, and the third root $z_{3}$ of multiplicity $k$, moves along the perpendicular bisector of the segment $[0,1]$. Then for the critical points of a polynomial $P(z)$, which does not coincide with the points $z_{1}, z_{2}$ and $z_{3}$ we have:

a) The geometric locus of such critical points of $P(z)$, for $z_{3}$ such that $\left|\operatorname{Im} z_{3}\right| \leq \frac{\sqrt{k^{2}+2 m k}}{2 m}$, is the circle

$$
\left(x-\frac{1}{2}\right)^{2}+y^{2}=\left(\frac{\sqrt{k^{2}+2 k m}}{2(2 m+k)}\right)^{2}
$$

which intersection with a straight line $y=\frac{m \cdot \operatorname{Im} z_{3}}{2 m+k}$ determines the location of prime critical points of $P(z)$.

b) For $z_{3}$ such that $\left|\operatorname{Im} z_{3}\right|>\frac{\sqrt{k^{2}+2 m k}}{2 m}$, the geometric locus of critical points of $P(z)$ is the perpendicular bisector of the segment $[0,1]$. Which intersection with a circle, the center of which divides the segment $\left|z_{1}-z_{3}\right|$ in the ratio of $m: 2 m+k$ measured from the point of $z_{1}$, with a radius equal to $\frac{m \cdot\left|z_{1}-z_{3}\right|}{2 m+k}$ determine the location of such critical points of $P(z)$.

Proof. Let $P(z)=z^{m}(z-1)^{m}\left(z-\left(\frac{1}{2}+i y_{3}\right)\right)^{k}$, then the critical points of polynomial $P(z)$, which does not coincide with the roots $z_{1}, z_{2}$ and $z_{3}$ is the solution of equation

$(2 m+k) z^{2}-\left(2 m+k+2 m i y_{3}\right) z+\left(\frac{1}{2}+i y_{3}\right) m=0$

Solving this equation, with the condition $\left|y_{3}\right| \leq \frac{\sqrt{k^{2}+2 m k}}{2 m}$, we get

$$
\xi=\frac{1}{2} \pm \frac{\sqrt{k^{2}+2 m k-4 m^{2} y_{3}^{2}}}{2(2 m+k)}+i \frac{m y_{3}}{2 m+k},
$$

hence $\left|\xi-\frac{1}{2}\right|=\sqrt{\frac{k^{2}+2 k m}{2(2 m+k)}}$ and $\operatorname{Im} \xi=\frac{m \cdot y_{3}}{2 m+k}$, which proves the statement a) of this theorem.

b) In the case of $\left|y_{3}\right|>\frac{\sqrt{k^{2}+2 m k}}{2 m}$

$$
\xi=\frac{1}{2}+i\left(\frac{m \cdot y_{3}}{2 m+k} \pm \frac{\sqrt{4 m^{2} y_{3}^{2}-2 m k-k^{2}}}{2(2 m+k)}\right)
$$


hence $\operatorname{Re} \xi=\frac{1}{2}$ and

$\left|\xi-\left(\frac{m}{2(2 m+k)}+i \frac{m y_{3}}{2 m+k}\right)\right|=$

$\sqrt{\frac{m^{2}+2 m k-2 m}{4(2 m+k)^{2}}+\frac{m^{2} y_{3}^{2}}{(2 m+k)^{2}}}=\frac{m\left|z_{1}-z_{3}\right|}{2 m+k}$

Which proves the statement $b$ ) of this theorem.

Remark 3. Theorems 1-6 can be formulated for an arbitrary interval.

\section{Conclusion}

In the paper we consider the dynamic behavior of the critical points of some cubic polynomials, with the motion of one of the roots of the polynomial along a given trajectory.

Given problem has not been studied before and it is of interest a more general formulation of this problem in the case of polynomials of general form.

\section{REFERENCES}

[1] Prasolov, V.V, Polynomials, Springer, 2000.

[2] Rahman, Q.I and Schmeisser, G., Analytic theory of polynomials, Oxford Univ. Press, 2005.

[3] Marden M, Geometry of Polynomials, AMS, 1966.

[4] Schmeisser, G., The conjectures of Sendov and Smale, Approximation Theory (a volume dedicated to Blagovest Sendov), Sofia Darba, 2002, pp 353-369.

[5] Smale S., The fundamental theorem of algebra and complexity theory, Bulletin of AMS 4(1981), pp. 1-36.

[6] Sendov B1., Generalization of a conjecture in the Geometry of Polynomials, Serdica Math. J. 28(2002), pp.283-304.

[7] Kalman, D., An Elementary Proof of Marden's Theorem, The American Mathematical Monthly, vol.115, no. 4, 2008, pp.330-338.

[8] Linfield, B. Z., On the relation of the roots and poles of a rational function to the roots of its derivative, Bulletin of the AMS, 27(1920), pp. 17-21. 